\title{
Minimally invasive surfactant therapy versus InSurE in preterm neonates of 28 to 34 weeks with respiratory distress syndrome on non-invasive positive pressure ventilation-a randomized controlled trial
}

\author{
Bhupendra Kumar Gupta ${ }^{1}$ - Anindya Kumar Saha ${ }^{1}$ - Suchandra Mukherjee ${ }^{1}$ Bijan Saha ${ }^{1}$ \\ Received: 24 March 2020 / Revised: 7 May 2020 / Accepted: 9 May 2020 / Published online: 27 May 2020 \\ (C) Springer-Verlag GmbH Germany, part of Springer Nature 2020
}

\begin{abstract}
Preterm neonates with respiratory distress syndrome (RDS) are commonly treated with surfactant by intubate surfactant extubate (InSurE) technique. Mode of surfactant administration has evolved towards less invasive technique in the last few years. We randomised 58 preterm infants of 28-34 weeks of gestation with RDS within $6 \mathrm{~h}$ of birth to receive surfactant by InSurE or minimally invasive surfactant therapy (MIST). Non-invasive positive pressure ventilation (NIPPV) was used as primary respiratory support. The main objective was to compare the need of invasive mechanical ventilation (IMV) in first $72 \mathrm{~h}$ of life and secondarily hemodynamically significant patent ductus arteriosus (hsPDA), intraventricular haemorrhage (IVH) (> grade 2), bronchopulmonary dysplasia (BPD) and composite outcome of BPD/mortality. We did not find any difference in need of IMV in first $72 \mathrm{~h}$ between MIST and InSurE (relative risk with MIST, $0.62 ; 95 \%$ confidence interval, 0.22 to 1.32 ). No difference was observed in terms of hs PDA, IVH (> grade 2), BPD and composite outcome of $\mathrm{BPD} /$ mortality.

Conclusion: There is no difference between MIST and InSurE in preterm neonates with RDS with NIPPV as a primary mode of respiratory support. Larger multicentre studies are needed to further explore differences in treatment failure and other secondary outcomes.
\end{abstract}

Trial registration: www.ctri.nic.in id CTRI/2019/03/017992, registration date March 8, 2019.

What is Known

- InSurE is commonly used for many years for treatment of RDS in preterm neonates.

- MIST has been introduced as a newer tool.

What is New

- MIST with feeding tube is comparable with InSurE in preterm infants with RDS in developing countries.

-NIPPV can be used as primary respiratory support for MIST.

Keywords InSurE $\cdot$ MIST $\cdot$ RDS $\cdot$ Preterm $\cdot$ NIPPV $\cdot$ Neonates

See related article, https://doi.org/10.1007/s00431-020-03688-3

Communicated by Daniele De Luca

Bijan Saha

bijansaha18@gmail.com

Bhupendra Kumar Gupta

bhugup@gmail.com

Anindya Kumar Saha

sahaanindya09@gmail.com

Suchandra Mukherjee

drsmukherjee70@gmail.com

Extended author information available on the last page of the article

$\begin{array}{ll}\text { Abbreviations } \\ \text { BPD } & \text { Bronchopulmonary dysplasia } \\ \text { CI } & \text { Confidence interval } \\ \text { DPPC } & \text { Dipalmitoyl phosphatidyl choline } \\ \text { FRC } & \text { Functional residual capacity } \\ \mathrm{FiO}_{2} & \text { Fraction of oxygen in inspired air } \\ \text { hs PDA } & \text { Hemodynamically significant patent } \\ & \begin{array}{l}\text { ductus arteriosus } \\ \text { IMV }\end{array} \\ \text { InSurE } & \text { Intubate surfactant extubate } \\ \text { IVH } & \text { Intraventricular haemorrhage } \\ \text { MIST } & \text { Minimally invasive surfactant therapy } \\ \text { nCPAP } & \text { Nasal continuous positive airway pressure }\end{array}$




$\begin{array}{ll}\text { NIPPV } & \text { Non-invasive positive pressure ventilation } \\ \text { NICU } & \text { Neonatal intensive care unit } \\ \text { PEEP } & \text { Positive end expiratory pressure } \\ \text { PIP } & \text { Peak inspiratory pressure } \\ \text { RR } & \text { Relative risk }\end{array}$

\section{Introduction}

Preterm babies $(<34$ weeks of gestation) with respiratory distress syndrome managed on non-invasive ventilation require surfactant administration via endotracheal tube [1].Time-tested InSurE technique requires intubation of the trachea and positive pressure ventilation. Newer minimally invasive strategies for surfactant therapy (MIST) are being increasingly used to manage RDS $[1,2]$. Various studies have shown the benefits and feasibility of MIST in treating preterm infants with RDS, mostly in high-income developed countries $[1,3]$.

Application of nasal continuous positive airway pressure (nCPAP) is a standard practice for babies managed with InSurE. Across the studies, the incidence of nCPAP failure rate in the first 7 days of life is 46 to $51 \%$ [4-6] and nCPAP failure is linked to increased use of invasive mechanical ventilation (IMV). Instead, nasal intermittent positive pressure ventilation (NIPPV) can be a better alternative as it can deliver time-cycled positive pressure ventilation above positive end expiratory pressure (PEEP) level in the absence of an endotracheal tube. NIPPV as primary respiratory support has lower rate of respiratory failure and need for intubation within the first week of life [7-10].However, there is paucity of data regarding feasibility and efficacy of MIST in the developing countries and to the best of our knowledge, none of the previous studies compared MIST with InSurE keeping NIPPV as the primary mode of respiratory support. We planned this randomised trial to compare the efficacy of MIST and InSurE technique on NIPPV as primary respiratory support among infants with RDS with gestational age of 28 to 34 weeks.

\section{Material and methods}

\section{Trial design, settings and participants}

This randomised trial was conducted in level III neonatal intensive care unit (NICU) in a tertiary care hospital in Kolkata, India from March 2019 to December 2019. Infants with gestation age of 28 to 34 weeks diagnosed with RDS were enrolled in the study. Infants with major congenital anomalies, perinatal asphyxia and those who required intubation at labour room were excluded.

\section{Intervention}

In all the infants admitted in the NICU with respiratory distress since birth, NIPPV was started with initial settings of peak inspiratory pressure (PIP) of $12-15 \mathrm{~cm}$ of $\mathrm{H}_{2} \mathrm{O}$, PEEP of 5-6 $\mathrm{cm}$ of $\mathrm{H}_{2} \mathrm{O}$, rate of 40 min and fraction of oxygen in inspired air $\left(\mathrm{FiO}_{2}\right)$ adjusted to achieve a target saturation of 90 to $95 \%$ by using the Drager babylog 8000 plus ventilator. Short binasal prongs or mask along with nasal tubing were used as interface for NIPPV. RDS was diagnosed clinically in preterm infants by a clinical team in the NICU based on the need of supplemental oxygen, clinical signs of tachypnea, retraction and grunting and with suggestive chest $\mathrm{x}$-ray done immediately. Patients who required $\mathrm{FiO}_{2}$ more than $30 \%$ on NIPPV to maintain saturation $\left(\mathrm{SpO}_{2}\right)$ between 90 and $95 \%$ in first $6 \mathrm{~h}$ of life were randomised to receive surfactant either by MIST or InSurE technique.

\section{MIST procedure}

The procedure was performed in the NICU by two trained neonatologists and a staff nurse for documentation and for assistance if required any. Prior to MIST procedure, interface was changed to snugly fitting nasal cannula for delivering NIPPV and infant was positioned in sniffing position. Heart rate and $\mathrm{SpO}_{2}$ were monitored throughout the procedure. Direct laryngoscopy was performed and a $5 \mathrm{Fr}$ feeding tube was inserted to the desired depth with Magill forceps. The required tip to lip length was calculated as weight in kilograms plus $7 \mathrm{~cm}$ as per our local NICU method. In a study conducted by Dragaville PA et al. [11], desired depth of insertion beyond the vocal cord was $1 \mathrm{~cm}$ for $25-26$ weeks, $1.5 \mathrm{~cm}$ for $27-28$ weeks and $2 \mathrm{~cm}$ for 29-32 weeks. Due to technical difficulty in visualising the exact length of the feeding tube beyond the vocal cord, we tried to get the tip to lip length for MIST.

No sedation or premedication was used but nesting and swaddling were done during the procedure for the comfort of the baby. After feeding tube placement, the laryngoscope was removed. Poractant alpha (Curosurf, Chiesi Farmaceutici Group, Parma, Italy) at the dose of $200 \mathrm{mg} / \mathrm{kg}$ was used for surfactant replacement therapy.The surfactant was drawn up in a 5- or 10-ml syringe and given in 1-ml aliquot in stages with each stage lasting for $10 \mathrm{~s}$. After complete dose administration, the feeding tube was withdrawn. Following the MIST procedure, the nasal interface was changed again to standard nasal prong/mask and nasal tubing for NIPPV. For infants who had desaturation with $\mathrm{SpO}_{2}$ less than $85 \%$, $\mathrm{FiO}_{2}$ was escalated in increment of 5\%. If the infant had apnoea lasting more than $20 \mathrm{~s}$, then positive pressure ventilation was initiated as per protocol of the NICU. If the infant continued to require $\mathrm{FiO}_{2}$ more than $30 \%$ after $6 \mathrm{~h}$ of initial surfactant therapy, the second dose of surfactant was given by the same technique. 
Failure on NIPPV was considered when there was requirement of intubation due to presence of persistent respiratory acidosis with $\mathrm{pH}<7.2$ and $\mathrm{pCO}_{2}$ more than $60 \mathrm{~mm} \mathrm{Hg}$ or recurrent apnoea requiring positive pressure ventilation or requiring NIPPV setting of $\mathrm{FiO}_{2}>60 \%$, PIP more than $25 \mathrm{~cm}$ of $\mathrm{H}_{2} \mathrm{O}$ and PEEP more than $6 \mathrm{~cm}$ of $\mathrm{H}_{2} \mathrm{O}$.

For de-escalation of respiratory support, infants were weaned to nCPAP when they showed minimal or no signs of respiratory distress and were apnoea free for at least $24 \mathrm{~h}$ with NIPPV setting of PIP $12-14 \mathrm{~cm}$ of $\mathrm{H}_{2} \mathrm{O}$, PEEP $4 \mathrm{~cm}$ of $\mathrm{H}_{2} \mathrm{O}$ and $\mathrm{FiO}_{2}$ less than $25 \%$. Infants were weaned from nCPAP as per unit protocol.

\section{InSurE procedure}

In InSurE technique, infants were intubated with appropriate size endotracheal tube. No sedation or premedication was used but nesting and swaddling were done during procedure. For surfactant replacement therapy, poractant alpha (Curosurf, Chiesi Farmaceutici Group, Parma, Italy) (200 mg/kg) was administered with positive pressure ventilation with appropriate size selfinflating resuscitation bag. Babies were extubated immediately after few minutes of surfactant administration and after extubation, infants were put on NIPPV again. The criteria for subsequent doses of surfactant, requirement of intubation as failure and weaning were same as in the MIST group.

\section{Outcomes}

The primary outcome of the study was the need of IMV in the first $72 \mathrm{~h}$ of life. The secondary outcomes which were studied were incidence of hsPDA [12], IVH > grade 2 [13], BPD (as defined by Jobe and Bancalari 2001) [14] and composite outcome of BPD/mortality before discharge. Duration of hospital stay was also studied as secondary outcome.

\section{Sample size}

Previous retrospective data from the study in the NICU and one previous study [15] showed that almost $55 \%$ of the infants in the InSurE group require intubation in the first $72 \mathrm{~h}$ of life. To reduce the need of IMV with MIST to $20 \%$ with alpha error 0.05 and power of $80 \%$, we estimated a sample size of 29 in each group.

\section{Randomisation}

Randomisation was done by computer-generated random sequence number. The allocation ratio was 1:1 and concealment was done by using a serially numbered opaque sealed envelope. The generation of random numbers and assignment was done by a person not involved in the study. However, the blinding of the intervention was not performed in any stages of the study from intervention to assessing the outcomes and data analysis due to the nature of the treatment.

\section{Statistical analysis}

The data analysis was done by Statistica version 6 (Tulsa, OK: StatSoft Inc., 2001) and MedCalc version 11.6 (Mariakerke, Belgium: MedCalc Software 2011). Data was summarized by routine descriptive statistics, namely mean and standard deviation for numerical variables that were normally distributed, median and interquartile range for skewed numerical variables, and counts and percentages for categorical variables. Numerical variables were compared between subgroups by Student's independent samples $t$ test, if normally distributed, or by Mann-Whitney $U$ test, if otherwise. Fisher's exact test or Pearson's chi-square test was employed along with calculation of relative risk (RR) and $95 \%$ confidence interval (CI) for intergroup comparison of categorical variables. Analyses were two-tailed and statistical significance level was set at $p<0.05$ for all comparisons.

\section{Ethics}

This study was prospectively approved by institutional ethics committee of the Institute of Post Graduate Medical Education and Research, Kolkata, India. Written informed consent was obtained from all legal guardians before participation in the study. This trial was registered in clinical trial registry of India (registration number CTRI/2019/03/017992).

\section{Result}

A total of 58 infants were randomised to one of the two groups of MIST and InSurE (Fig. 1). Both groups were comparable with the baseline variables (Table 1). The average gestation age of the infants was 30 weeks whereas the average birth weight of the population was $1223 \mathrm{~g}$. We observed a high proportion of caesarean section delivery (55-65\%). Average time of requirement of surfactant from birth was $1 \mathrm{~h}$. The mean duration of surfactant administration and bagging was recorded as $182 \mathrm{~s}$ (sd 7.59).

The results of primary and secondary outcomes are depicted in Table 2. There was no statistically significant difference in need of IMV in $72 \mathrm{~h}$ of life between the MIST group (10.34\%) and the InSurE group (20.69\%) (relative risk [RR] with MIST, $0.62 ; 95 \%$ confidence interval $[\mathrm{CI}], 0.22$ to 1.32 ). No differences were observed between the two groups for hsPDA, IVH $>$ grade 2, BPD and composite outcome of BPD and mortality before discharge. Infants in the InSurE group stayed in the hospital for more days in comparison with the MIST group (mean 41.6 days (sd 23.11) vs mean 29.76 days (sd 18.09); $p$ 0.03). We did not find any difference in the requirement of second dose of surfactant between the two groups. 
Fig. 1 CONSORT flow chart

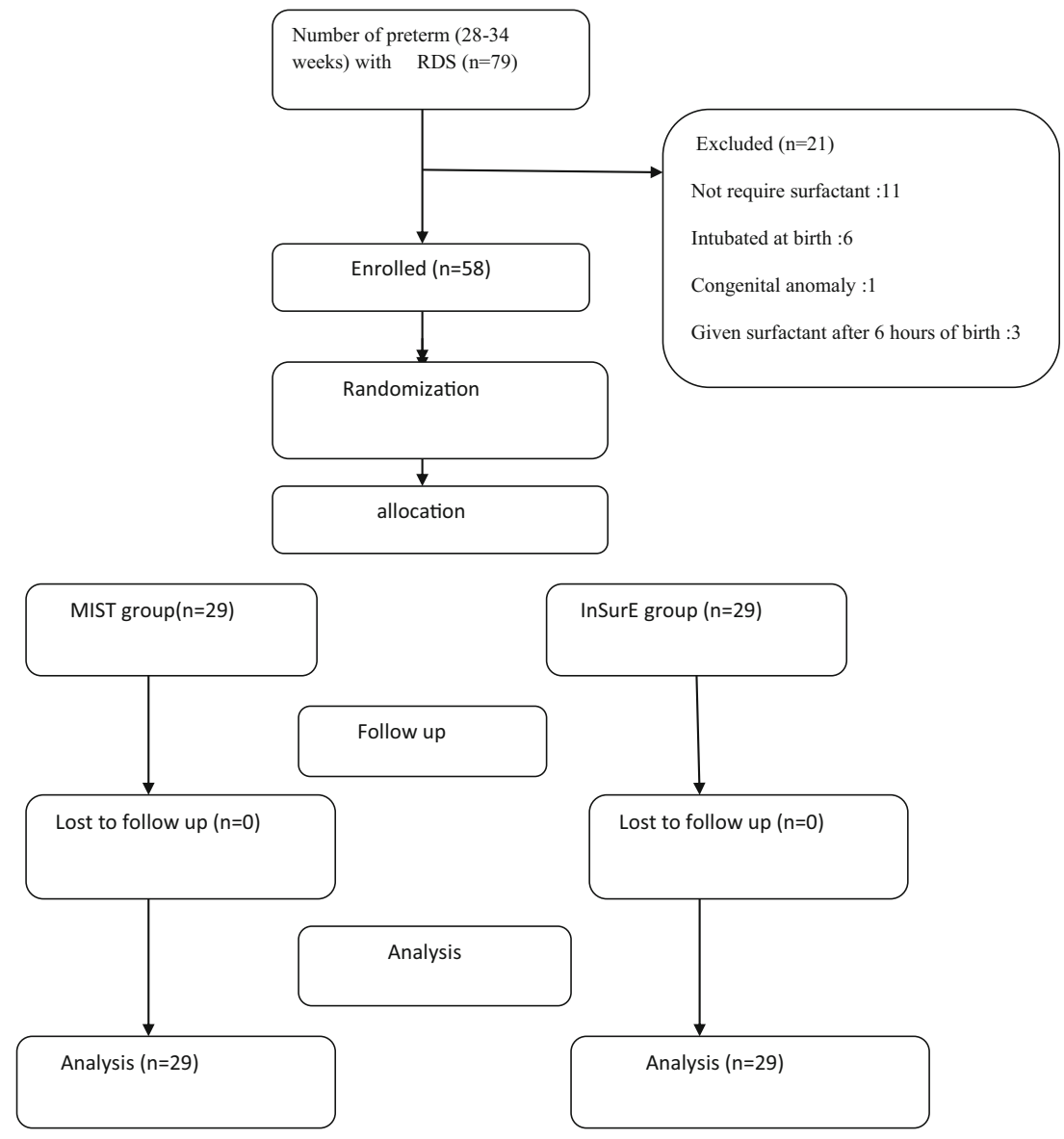

\section{Discussion}

In this randomised control trial, we compared efficacy of MIST with InSurE in infants of 28 to 34 weeks in RDS using NIPPV as a primary respiratory support. We found there was no difference of need of IMV in MIST in comparison with
InSurE if we use NIPPV in preterm RDS.There was also no difference in composite outcome of BPD/mortality (RR with MIST, $0.55 ; 95 \%$ CI, 0.22 to 1.22) and BPD among survivors (RR with MIST $0.61 ; 95 \% \mathrm{CI}, 0.24$ to 1.22 ) between two groups. Decreased need of IMV in the MIST group compared with the InSurE group was reported in a recent study [16]. The
Table 1 Baseline variables of the enrolled subjects

\begin{tabular}{llll}
\hline Variable & MIST $(n=29)$ & InSurE $(n=29)$ & $p$ value \\
\hline Gestation age, weeks mean (sd) & $30.07(1.51)$ & $29.90(1.67)$ & 0.68 \\
Birth weight, g, mean (sd) & $1225(281)$ & $1222(322)$ & 0.97 \\
Male, $n(\%)$ & $18(62.07)$ & $18(62.07)$ & 1 \\
Antenatal steroid (any dose) $n(\%)$ & $23(79.31)$ & $24(82.76)$ & 1 \\
Antenatal steroid (complete course with 4 doses), $n(\%)$ & $1(3.44)$ & $3(10.340$ & 0.61 \\
Multigravida, $n(\%)$ & $6(20.68)$ & $6(20.68)$ & 1 \\
Caesarean section delivery, $n(\%)$ & $19(65.52)$ & $16(55.17)$ & 0.59 \\
Apgar 5 min (1-10), median (IQR) & $8(7-9)$ & $8(7-9)$ & 0.24 \\
FiO2 before surfactant therapy, $\%$, median (IQR) & $50(35-60)$ & $45(40-55)$ & 0.59 \\
Time of birth to surfactant therapy, h, median (IQR) & $1(1-2)$ & $1(1-2)$ & 0.82 \\
$\mathrm{CRIB}_{-1 \mathrm{II}}$ scores, mean (sd) & $6.24(2.66)$ & $6.31(2.48)$ & 0.91 \\
$\mathrm{SpO}_{2}$ /FiO 2 ratio before surfactant, median (IQR) & $186(184-206)$ & $202(184-204)$ & 0.90 \\
\hline
\end{tabular}


Table 2 Outcome variables of the two groups-MIST and InSurE

\begin{tabular}{|c|c|c|c|c|}
\hline Variable & $\operatorname{MIST}(n=29)$ & $\operatorname{InSurE}(n=29)$ & $\begin{array}{l}\text { Relative risk } \\
\text { ( } 95 \% \text { confidence interval) }\end{array}$ & $p$ value \\
\hline Invasive mechanical ventilation, $n(\%)$ & $3(10.34)$ & $6(20.69)$ & $0.62(0.22-1.32)$ & 0.47 \\
\hline Hemodynamically significant patent ductus arteriosus, $n(\%)$ & $6(20.69)$ & $8(27.58)$ & $0.82(0.39-1.45)$ & 0.76 \\
\hline Intraventricular haemorrhage $>$ grade $2, n(\%)$ & $0(0)$ & $1(3.45)$ & $0.00(0.00-1.64)$ & $>0.99$ \\
\hline Bronchopulmonary dysplasia, $n(\%)$ & $4(13.79)$ & $8(27.59)$ & $0.61(0.24-1.22)$ & 0.17 \\
\hline Bronchopulmonary dysplasia/mortality before discharge, $n(\%)$ & $4(13.79)$ & $9(31.03)$ & $0.55(0.22-1.12)$ & 0.11 \\
\hline Second dose of surfactant, $n(\%)$ & $3(10.3)$ & $2(6.89)$ & $1.22(0.45-2.08)$ & $>0.99$ \\
\hline
\end{tabular}

use of MIST was also found to reduce the composite outcome of death or BPD and need for IMV within $72 \mathrm{~h}$ of birth in various recent meta-analyses [17, 18].

But in our study, NIPPV was used as a primary mode of respiratory support where as in most of the previous studies with MIST, nCPAP was the primary mode of respiratory support $[1,3]$. This might have reduced the need of IMV in both groups in our studies. There is already an evidence in literature that supports NIPPV as primary mode of respiratory support to decrease the need of IMV [7]. The side-effects of MIST related to airflow limitation and increased resistance can be overcome in adults by using non-invasive ventilation [19], which has also been suggested as being beneficial for preterm neonates undergoing MIST [20]. Even in a physiological study, it has been shown NIPPV may overcome the leaks and tracheal obstruction due to catheterisation with MIST catheter. This may allow some pressure delivery down the alveoli which is not transmitted if nCPAP is used during MIST [21].

Failure to insert the catheter through the vocal cords at first attempt, significant surfactant reflux, acute desaturations, bradycardia and/or need for manual ventilation during surfactant administration through tracheal feeding tube were observed in $<10 \%$ [22] to $>30 \%$ [1] in different studies behind the unsuccessful attempts of MIST. Interruption of nCPAP during the procedure was the problem in previous studies; we kept continuous NIPPV support through appropriate nasal interface during the MIST manipulation. It minimised incidence of desaturation, bradycardia and need of manual ventilation here during MIST.There was also 96.5\% success rate for the first attempt at introduction of feeding tube in MIST technique in the present study whereas it was reported as $75 \%$ in one previous study [23]. High success rate in our study could be due to training of neonatologists in MIST technique prior to recruitment of the study.

In our study, the requirement of second dose of surfactant was small. There was also very negligible incidence of surfactant reflux $(<10 \%)$ during the MIST procedure in our study. Three babies in the InSurE group and two babies in the MIST group required second dose of surfactant. There was no statistical difference in requirement in second dose surfactant between the two groups. In the study conducted by M Aguar et al. [24], second dose surfactant was required more in the MIST group than the InSurE (35.6\% vs $6.5 \%, p=0.003)$ possibly due to use of smaller dose of surfactant in the MIST procedure $(100 \mathrm{mg} / \mathrm{kg})$ compared with InSurE (200 $\mathrm{mg} / \mathrm{kg}$ ). But similar dose of $200 \mathrm{mg} / \mathrm{kg}$ of poractant alpha was used in both groups in the present study. This higher incidence of surfactant retreatment in MIST [24] may also be due to the fact that MIST does not provide any pressure to help surfactant spreading while InSure does $[25,26]$. But continuous NIPPV support during MIST in our study may have some better pressure effect which reduces the need of surfactant retreatment in our study.

In the present study, infants in the MIST group also showed less hospital stay. The difference was also statistically significant and this has major economic and social impact in background of the low socioeconomic condition. But this outcome is well known for its extreme variability and it was also not a trial prespecified outcome. It is significantly influenced by many other factors such as complications occurring during NICU stay (sepsis, growth and feeding problems), social factors (parental presence and efficacy) and public health factors (availability of beds, existence of back transfer protocols, second level hospital, etc.). Until those factors are not considered in well-powered trials, these outcomes should not be taken seriously for implementation of MIST as a standard therapy. Hence, it is extremely unlikely that this result may be related to an intervention performed in the first day of life and should not be regarded as such.

The biological plausibility of seemingly better distribution of surfactant in spontaneously breathing infants and less risk of airway injury in MIST technique have been challenged in the view point by De Luca D [25]. Some of the main pathophysiological problems of MIST have not been taken into account in the present study which can be considered as limitations of this study. We did not use any premedication for sedation before MIST or InSurE and used only non-pharmacological method like nesting and swaddling for the baby's comfort .The lack of sedation for both interventions may present ethical problems in 
some countries. InSurE in our study was done by bagging rather than with pressure limited volume guarantee ventilation by ventilator. This may introduce a bias of probable lung injury [25]. There was no blinding in the study and even the outcome assessors were not blinded. The infants with gestation less than 28 weeks were not included. In the sample size calculation, the baseline intubation rate was determined mainly from the personal experience based on some retrospective data from the NICU and not directly from any robust previous clinical trial of similar population. The sample size was also small with low post hoc calculated power. All these can produce bias in the results.

In our study, we used different interfaces like nasal mask or prong in a single baby as per our NICU protocol to reduce trauma, which may influence variation in leaks. These may impact on the efficacy of NIPPV as some of them were suboptimal. Moreover, the use of such different interfaces cannot reduce the leak and improve pressure transmission while the mouth is open, even if NIPPV is used [27]. We have used a low threshold of $30 \% \mathrm{FiO} 2$ for indication of surfactant therapy instead of a higher one which may have some bearing on the results. Furthermore, the retreatment of surfactant at $6 \mathrm{~h}$ after the initial dose may be considered early as the median half-life of dipalmitoyl phosphatidylcholine (DPPC) in preterm babies is $10-11 \mathrm{~h}$ as found in a physiological study [28]. However, surfactant retreatment can be done earliest at 2-6 h of first dose as found in clinical study and published guidelines [29, 30] and hence it has been used as our NICU protocol. It is also found that our study population had fast worsening RDS requiring $40-50 \% \mathrm{FiO} 2$ within 1-2 h of life and also had a very high occurrence of combined outcome of BPD/mortality in contrast with the reports from other centres in developed countries. This can be related with very poor coverage of complete course of antenatal steroid coverage in our population which actually may make translation of the results in other setting difficult. We could not attempt multivariate analysis for better evaluation of risk factors of $\mathrm{BPD} /$ mortality because we did not have significantly different basic variables between two study arms. There is a need for larger adequately powered trial of MIST vs InSurE considering all these and with NIPPV as a primary respiratory support.

To conclude, this study has shown that there is no difference between MIST and InSurE in preterm neonates with RDS with NIPPV as a primary mode of respiratory support. To the best of our knowledge, this is the first study of MIST vs InSurE on preterm infants on NIPPV.

Authors' contributions Dr. Bhupendra Kumar Gupta conceptualised and designed the study, developed the protocol, patient management, analyzed the data, and prepared the first draft.

Dr. Anindya kumar Saha, critically reviewed, revised the manuscript, and helped in statistical analysis of results.

Dr. Suchandra Mukherjee helped in protocol development and critically reviewed the manuscript for improving the content.
Dr. Bijan Saha helped in protocol development, co-ordinated, supervised data collection, reviewed and revised the manuscript at all stages of its production.

All the authors approved the final manuscript as submitted and agree to be accountable for all aspect of the work.

\section{Compliance with ethical standards}

Conflict of interest The authors declare that they have no conflict of interest.

Ethics approval The ethical approval for the study was taken from the Institutional Ethics Committee, Institute of Post Graduate Medical Education and Research,, Kolkata, India(Inst/IEC/2018/393, dated 28.04.2019).

Informed consent to participate Informed consent was obtained from the legal guardian of all included participants.

\section{References}

1. Dargaville PA, Ali SKM, Jackson HD, Williams C, De Paoli AG (2016) Impact of minimally invasive surfactant therapy in preterm infants at 29-32 weeks gestation. Neonatology 113:7-14

2. Verder H, Robertson B, Greisen G et al (1994) Surfactant therapy and nasal continuous positive pressure for newborn with respiratory distress syndrome. N Engl J Med 331(16):1051-1055

3. Kribs A, Pillekamp F, Hunseler C, Vierzig A, Roth B (2007) Early administration of surfactant in spontaneous breathing with nCPAP: feasibility and outcome in extremely premature infants (postmenstrual age $\leq 27$ weeks). Paediatr Anaesth 17(4):364-369

4. Morley CJ, Davis PG, Doyle LW, Brion LP, Hascoet JM, Carlin JB (2008) Nasal CPAP or intubation at birth for very preterm infants. N Engl J Med 358:700-708

5. Finer NN, Carlo WA, Walsh MC et al (2010) Early CPAP versus surfactant in extremely preterm infants. N Engl J Med 362:1970 1979

6. Dunn MS, Kaempf J, de Klerk A et al (2011) Randomised trial comparing 3 approaches to the initial respiratory management of preterm neonates. Pediatrics 128:e1069-e1076

7. Behnke J, Lemyre B, Czernik C, Zimmer KP, Ehrhardt H, Waitz M (2019) Non-invasive ventilation in neonatology. DeutschesArztebla International 116(11):177-183

8. Lemyre B, Laughon M, Bose C, Davis PG (2016) Early nasal intermittent positive pressure ventilation (NIPPV) versus early nasal continuous positive pressure(nCPAP) for preterm infants. Cochrane Database Syst Rev 2:CD003212

9. Kishore MSS, Dutta S, Praveen K (2009) Early nasal intermittent positive pressure ventilation versus nasal continuous positive airway pressure for respiratory distress syndrome. Actapaediatrica 98: 1412-1415

10. Shi Y, Tang S, Zhao J, Shen J (2013) A prospective ,randomised, controlled study of NIPPV versus nCPAP in preterm and term infants with respiratory distress syndrome. Pediatr Pulmonol 49:673678

11. Dargaville PA, Aiyappan A, De Paoli AG, Kuschel CA et al (2013) Minimally invasive surfactant therapy in preterm infants on continuous positive airway pressure. Arch Dis Child Fetal Neonatal 98: F122-F126

12. Sehgal A, McNamara PJ (2009) Does echocardiography facilitate determination of hemodynamically significance attributable to the ductus arteriosus ? Eur J Pediatr 168(8):907-914 
13. Papile LA, Burstein J,Brustein R, Koffler H. (1978) Incidence and evolution of subependymal and intraventricular hemorrhage: a study of infants with birth weights less than 1,500gm.pediatrics 92:529-34

14. Jobe AH, Bancalari E (2001) Bronchopulmonary dysplasia: NICHD/NHLBI/ORD workshop summary. Am J Respir Crit Care Med.163:1723-9

15. Kripalani H, Miller D, Lemyre B, Yoder BA, Chiu A, Roberts RS (2013) A trial comparing noninvasive ventilation strategies in preterm infants. N Engl J Med 369:611-620

16. Kanmaz HG, Erdeve O, Canpolat FE, Mutlu B, Dilmen U (2013) Surfactant administration via thin catheter during spontaneous breathing: randomised controlled trial. Pediatrics 131(2):e502-e509

17. Lau CSM, Chamberlain RS, Sun S (2017) Less invasive surfactant administration reduces the need for mechanical ventilation in preterm infants: a meta-analysis. Global Pediatric Health 4:1-9

18. Aldana-Aguirre JC, Pinto M, Featherstone RM, Kumar M (2017) Less invasive surfactant administration versus intubation for surfactant delivery in preterm infants with respiratory distress syndrome : a systematic review and meta analysis. Arch Dis Child Fetal Neonatal Ed 102:F17-F23

19. Antonelli M, Conti G, Rocco M, Arcangeli A, Cavaliere F, Proietti R, Meduri GU (2002) Noninvasive positive-pressure ventilation vs conventional oxygen supplementation in hypoxemic patients undergoing diagnostic bronchoscopy. Chest 121:1149-1154

20. Oncel MY, Arayici S, Uras N, Alyamac-Dizdar E, Sari FN, Karahan S, Canpolat FE, Oguz SS, Dilmen U (2016) Nasal continuous positive airway pressure versus nasal intermittent positivepressure ventilation within the minimally invasive surfactant therapy approach inpreterm infants: a randomised controlled trial. Arch Dis Child Fetal Neonatal Ed 101:f323-f328

21. Jourdain G, De Tersant M, Dell'Orto V, Conti G, De Luca D (2018) Continuous positive airway pressure delivery during less invasive surfactant administration: a physiologic study. J Perinatol 38(3): 271-277

22. Göpel W, Kribs A, Ziegler A, Laux R, Hoehn T, Wieg C, Siegel J, Avenarius S, von der Wense A, Vochem M, Groneck P, Weller U, Möller J, Härtel C, Haller S, Roth B, Herting E, German Neonatal
Network (2011) Avoidance of mechanical ventilation by surfactant treatment of spontaneously breathing preterm infants (AMV): an open-label, randomised, controlled trial. Lancet 378:1627-1634

23. Mosayebi Z, Kadivar M, Derakhsh NT, Nariman S, Marashi SM, Farsi Z (2017) A randomized trial comparing surfactant administration using InSurE technique and the minimally invasive surfactant therapy in preterm infants (28 to 34 Weeks of Gestation) with Respiratory Distress Syndrome. J Compr Ped 8(4):e 60724

24. Aguar M, Cernada M, Brugada M, Gimeno A, Gutierrez A, Vento M (2014) Minimally invasive surfactant therapy with a gastric tube is as effective as the intubation, surfactant, and extubation technique in preterm babies. Acta Paediatr 103:e229-e233

25. De Luca D, Aguilera SS, Centorrino R, Fortas F, Yousef N, Carnielli VP (2020) Less invasive surfactant administration: a word of caution. Lancet Child Adolesc Health 4:331-340. https://doi.org/ 10.1016/S2352-4642(19)30405-5

26. Niemarkt HJ, Kuypers E, Jellema R, Ophelders D, Hütten M, Nikiforou M, Kribs A, Kramer BW (2014) Effects of lessinvasive surfactant administration on oxygenation, pulmonary surfactant distribution, and lung compliance in spontaneously breathing preterm lambs. Pediatr Res 76(2):166-170

27. Centorrino R, Dell'Orto V, Gitto E, Conti G, De Luca D (2019) Mechanics of nasal mask-delivered HFOV in neonates: a physiologic study. Pediatr Pulmonol 54(8):1304-1310

28. Cogo PE, Facco M, Simonato M, De Luca D, De Terlizi F, Rizzotti U, Verlato G, Bellagamba MP, Carnielli VP (2011) Pharmacokinetics and clinical predictors of surfactant redosing in respiratory distress syndrome. Intensive Care Med 37(3):510-517

29. Davis DJ, Barrington KJ (2005) Recommendations for neonatal surfactant therapy. Paediatr Child Health 10:109-116

30. Figueras-Aloy J, Quero J, Carbonell-Estrany X, et al.(2001) Early administration of the second dose of surfactant (beractant) in the treatment of severe hyaline membrane disease. Acta Paediatr 0.90: 296-301.

Publisher's note Springer Nature remains neutral with regard to jurisdictional claims in published maps and institutional affiliations.

\section{Affiliations}

\section{Bhupendra Kumar Gupta ${ }^{1} \cdot$ Anindya Kumar Saha ${ }^{1} \cdot$ Suchandra Mukherjee ${ }^{1} \cdot$ Bijan Saha $^{1}$}

1 Department of Neonatology, Institute of Post Graduate Medical Education \& Research and SSKM Hospital, 244, A J C Bose Road, Kolkata 700020, India 